\title{
MetroMMC: Electron-Capture Spectrometry with Cryogenic Calorimeters for Science and Technology
}

\author{
P. C.-O. Ranitzsch, et al. [full author details at the end of the article]
}

Received: 19 July 2019 / Accepted: 22 November 2019 / Published online: 9 December 2019

(c) The Author(s) 2019

\begin{abstract}
Accurate decay data of radionuclides are necessary for many fields of science and technology, ranging from medicine and particle physics to metrology. However, data that are in use today are mostly based on measurements or theoretical calculation methods that are rather old. Recent measurements with cryogenic detectors and other methods show significant discrepancies to both older experimental data and theory in some cases. Moreover, the old results often suffer from large or underestimated uncertainties. This is in particular the case for electron-capture (EC) decays, where only a few selected radionuclides have ever been measured. To systematically address these shortcomings, the European metrology project MetroMMC aims at investigating six radionuclides decaying by EC. The nuclides are chosen to cover a wide range of atomic numbers $Z$, which results in a wide range of decay energies and includes different decay modes, such as pure EC or EC accompanied by $\gamma$-and/or $\beta^{+}$-transitions. These will be measured using metallic magnetic calorimeters (MMCs), cryogenic energy-dispersive detectors with high-energy resolution, low-energy threshold and high, adjustable stopping power that are well suited for measurements of the total decay energy and X-ray spectrometry. Within the MetroMMC project, these detectors are used to obtain X-ray emission intensities of external sources as well as fractional EC probabilities of sources embedded in a $4 \pi$ absorber. Experimentally determined nuclear and atomic data will be compared to state-of-the-art theoretical calculations which will be further developed within the project. This contribution introduces the MetroMMC project and in particular its experimental approach. The challenges in EC spectrometry are to adapt the detectors and the source preparation to the different decay channels and the wide energy range involved, while keeping the good resolution and especially the low-energy threshold to measure the EC from outer shells.
\end{abstract}

Keywords Electron-capture decay $\cdot$ Metallic magnetic calorimeter $\cdot$ Radionuclide metrology 


\section{Introduction}

Radionuclides that decay by electron capture (EC) play an important role in many fields such as nuclear medicine, nuclear waste disposal, geo- and cosmo-chronology (see, e.g., [1,2] and references therein) and even for testing the standard model of particle physics in various ways, e.g., in neutrino physics [3-6]. Activity standards of EC nuclides are being used to calibrate $\gamma$-ray or X-ray detectors which requires accurate knowledge of the photon emission intensities of the respective radionuclides. Several EC nuclides are being used in medicine or considered as candidates for medical applications, e.g., ${ }^{67} \mathrm{Ga}$ and ${ }^{125}$ I. Here, the electron and photon emission spectra matter, e.g., when investigating the interaction of ionizing radiation with human tissue. When using long-lived EC isotopes for dating techniques the corresponding half-lives must be known.

Most of the mentioned applications require accurate activity standards. When using EC nuclides as calibration sources this is obvious, since reference sources with well-defined properties are needed. Moreover, the determination of photon emission intensities is based on accurate activity standardization. Also, the half-lives of long-lived radionuclides are determined by means of activity measurements and mass spectrometry (via $T_{1 / 2}=\frac{\ln (2) \cdot N}{A}$, with $A$ being the activity and $N$ the number of nuclei).

Despite the wide field of applications, the EC process along with the subsequent atomic rearrangement is not yet satisfactorily understood and accurate decay data are missing in many cases. Key data are the fractional EC probabilities, but also atomic data such as fluorescence yields and emission probabilities for X-rays and Auger electrons are very important. In particular for pure EC radionuclides (e.g., ${ }^{55} \mathrm{Fe}$, ${ }^{41} \mathrm{Ca}$ ), the fractional EC probabilities are a prerequisite for activity standardization when applying the triple-to-double coincidence ratio (TDCR) method with liquid scintillation counters [7], which is one of the best-established techniques for such radionuclides.

In recent years, a few measurements of $\beta^{-}$-decay and EC conducted by means of cryogenic detectors have shown significant discrepancies to previous experimental and/or theoretical data [8-13], which underlines the need of further accurate measurements and improvement of theoretical models. Corresponding proposals led to two projects: The recently concluded MetroBeta [14] project, which was concerned with $\beta^{-}$-decay, and the currently ongoing project MetroMMC to scrutinize the more challenging EC decay. In both projects the Laboratoire National Henri Becquerel (LNE-LNHB), the Physikalisch-Technische Bundesanstalt (PTB) and Heidelberg University are developing and applying metallic magnetic calorimeters (MMCs) for high-resolution spectrometry.

Within the MetroMMC project, the goal is to investigate six radionuclides, that decay by EC, namely ${ }^{41} \mathrm{Ca},{ }^{54} \mathrm{Mn},{ }^{59} \mathrm{Ni},{ }^{65} \mathrm{Zn},{ }^{109} \mathrm{Cd}$ and ${ }^{125} \mathrm{I}$. The relative and-if possible-absolute EC probabilities will be determined for all nuclides and the X-ray emission probabilities for each nuclide except for ${ }^{41} \mathrm{Ca} .{ }^{41} \mathrm{Ca}$ is an exception because of its sparseness and the very limited amount available at PTB $(A \approx 500 \mathrm{~Bq})$, which is not enough to produce a source for X-ray measurements, which usually require $A>10 \mathrm{kBq}$. The experimental work is based on metallic magnetic calorimeters 
(MMCs), which are perfectly suited for the task at hand due to their good energy resolution and especially their linearity and dynamic energy range. The measurements are accompanied by state-of-the-art theoretical calculations following two complementary approaches by dedicated theory groups. One theoretical approach is based on the LNHB developed software BetaShape [15], that is successfully used to describe the shape of $\beta$-spectra, which will be extended to include the influence of the atomic shell on the EC process and the subsequent atomic relaxation [16]. The second approach is based on the Quanty code [17] that is used to describe core-level X-ray spectroscopy and has been extended to EC processes for neutrino mass determination in the framework of the ECHo project [18]. The details of the theoretical aspects will not be further covered in this experimental overview.

${ }^{41} \mathrm{Ca}$ and ${ }^{59} \mathrm{Ni}$ decay purely by $\mathrm{EC}$, while the four remaining nuclides are accompanied by $\gamma$-transitions and ${ }^{65} \mathrm{Zn}$ has a significant contribution from a $\beta^{+}$-decay. The nuclides were chosen both for scientific reasons and practical ones, e.g., that the material is available in the required amounts, has sufficiently low radioactive contaminants, has a sufficiently long half-life and is compatible with sample preparation techniques, that are established or require a reasonable amount of development. Scientifically, both ${ }^{41} \mathrm{Ca}$ and ${ }^{59} \mathrm{Ni}$ are used for dating of the solar system, meteorites and in geology and ${ }^{41} \mathrm{Ca}$ plays a role in safety of nuclear waste disposal. The other isotopes have more difficult decay schemes, which make both experimental design and theoretical description more challenging. This is also supported by the fact that the chosen nuclides cover a wide range of atomic number $Z$ and different decay natures, e.g., allowed, 2 nd forbidden etc. In addition ${ }^{125} \mathrm{I}$ is of interest because of its use in nuclear medicine.

\section{MMC Design Considerations}

Design and fabrication techniques of metallic magnetic calorimeters have become very diverse and reliable over the last decades [19-21]. The design process usually starts with a specific application in mind. In particular the absorber dimensions are defined by the application, since the required stopping power or efficiency is defined by the used material and the absorber dimensions. With the given experimental conditions, e.g., operating temperature, other design parameters, e.g., read-out coil or sensor geometry, can be used to find an optimal detector design. Usually the optimum is rather broad; therefore, the design can be slightly altered without losing much performance. This gives the flexibility to fabricate detectors for different applications in one fabrication run or use the detector slightly outside its optimum.

Within the MetroMMC project two different types of measurements are being pursued, namely calorimetric spectrometry of the EC decay, where the whole spectrum, independent of decay channel is measured, and X-ray spectrometry, where only the radiative transitions of the decays are of interest. These measurement types have very different implications on the detector design, that will be discussed in the following section. 
Table 1 Highest energetic de-excitations of the six MetroMMC nuclides, that can be completely contained in the absorber, and the required gold thicknesses to reach $99.99 \%$ absorption efficiency, determined through Monte-Carlo simulations in PENELOPE. Higher energetic transitions [e.g., $\gamma$-transitions of ${ }^{54} \mathrm{Mn}$ $(834.848 \mathrm{keV})$ and $\left.{ }^{65} \mathrm{Zn}(1115.539 \mathrm{keV})\right]$ cannot be contained, but their effect on the spectrum shape is considered

\begin{tabular}{llcc}
\hline Nuclide & $E_{\max }$ type & $E_{\max } / \mathrm{keV}[22]$ & Required thickness $/ \mu \mathrm{m}$ \\
\hline${ }^{41} \mathrm{Ca}$ & $\mathrm{X}$-ray & 3.6028 & 3 \\
${ }^{54} \mathrm{Mn}$ & $\mathrm{X}$-ray & 5.987 & 8 \\
${ }^{59} \mathrm{Ni}$ & $\mathrm{X}$-ray & 7.706 & 20 \\
${ }^{65} \mathrm{Zn}($ low $E$ ) & $\mathrm{X}$-ray & 8.9771 & 25 \\
${ }^{65} \mathrm{Zn}($ high $E$ ) & $\beta^{+}$ & 329.9 & 70 \\
${ }^{109} \mathrm{Cd}($ low $E)$ & $\mathrm{X}$-ray & 25.512 & 85 \\
${ }^{109} \mathrm{Cd}($ high $E)$ & $\gamma$ & 88.0336 & 350 \\
${ }^{125} \mathrm{I}$ & $\gamma$ & 35.4922 & 210 \\
\hline
\end{tabular}

\subsection{Calorimetric Spectrometry}

The cleanest way to determine the fractional EC probabilities from the different atomic shells, without relying much on theoretical calculations, is to measure the decay calorimetrically, i.e., the whole decay energy at once, apart from the energy of the neutrino. This can be achieved by embedding the radionuclide in a $4 \pi$ absorber that fully absorbs the energy released by the decay and attaching the absorber to the temperature sensor of the MMC.

De-excitations after an EC event can both emit photons (X-ray, i.e., radiative transitions) or electrons (Auger, Coster-Kronig and super Coster-Kronig, i.e., non-radiative transitions) and both types of transitions need to be taken into consideration when determining the absorber dimensions. Since photons have the longer range in matter, compared to electrons, usually it is enough to determine the absorption efficiency for the highest energetic photons from the decay. If EC is accompanied by $\beta^{+}$-decay or $\gamma$-transitions, these channels need to be included in the simulation as well. The interaction of the decay products was simulated for the nuclides in question with gold as absorber material, using the Monte-Carlo software PENELOPE, to determine the thickness of the material necessary for a stopping power of $99.99 \%$ with the results shown in Table 1.

The resulting minimal absorber thicknesses are used to define the absorber geometry to match the heat capacity requirements of detector designs of the precursory project MetroBeta, where five detector designs for $\beta$-spectrometry with end-point energies ranging from $\sim 70$ to $\sim 700 \mathrm{keV}$ are available and described in detail in [14]. Due to the use of gold as absorber material and the allowed heat capacity for the different detector sizes, the maximum photon energy that can be completely contained in the absorber is limited to approximately $E_{\gamma}<100 \mathrm{keV}$. Higher energetic photons still need to be considered for their effect on the shape of the spectrum, e.g., through Compton scattering. 
In contrast to continuous $\beta$-spectra, calorimetric EC spectra show mono-energetic lines approximately at the binding energies of the captured electrons. To allow the measurement of the low energetic lines of EC from higher atomic shells, the detector threshold is of utmost importance. While shells with binding energies $<20 \mathrm{eV}$ are out of reach for even the smallest of the current detector designs, the measurement of energies $>20 \mathrm{eV}$ might only be limited by the detector and absorber size to reach the required stopping power and the accompanying threshold defined by energy resolution, which scales with the detector size.

High-energetic photons or $\beta$-particles require extremely large absorbers or deposit no or only part of their initial energy. To include this effect, it will be necessary to complement the analysis by sound Monte-Carlo simulations.

Another option is to split the measurements into two parts: one with full stopping power (large absorber) for the highest energy transitions and one for the lower energy transitions (small absorber), while allowing some of the higher energetic particles to escape. Such a two staged approach is currently planned for ${ }^{65} \mathrm{Zn}$ and ${ }^{109} \mathrm{Cd}$.

In particular for ${ }^{109} \mathrm{Cd}$, the approach seems to be promising. The measurement with large absorbers will permit to determine the decay rate of the $88 \mathrm{keV} \gamma$-transition of the ${ }^{109 \mathrm{~m}} \mathrm{Ag}\left(T_{1 / 2}=39 \mathrm{~s}\right)$ isomer. Consequently, the activity inside the absorber can be determined, which is a great advantage since the considered source preparation techniques (see Sect. 3) are non-quantitative methods. In addition, the measurement will determine the number of $K$ capture events and hence, the absolute value of the fractional $K$ capture probability can be derived. But even in ideal conditions, the threshold of the detector will be on the same order as the $M$ capture energies. The second stage with a smaller absorber will then lead to better energy resolution and lower threshold and permit to determine probabilities of $M$ or possibly even $N$ capture relative to the $\mathrm{K}-\mathrm{EC}$ probability.

\subsection{X-Ray Spectrometry}

The second step toward accurate nuclear decay data of EC decays is the measurement of the X-ray emission probabilities. These will also be performed using MMCs but require an entirely different setup. X-ray spectrometry is a widely used technique with MMCs, but the uncertainty budgets for metrology applications require special care in the experimental preparation and characterization of the detection efficiency (see e.g., $[23,24])$.

Starting from the external X-ray source, its activity needs to be well known and traceable to primary activity determination methods, and a well-defined geometry and low self-absorption are essential. In our case, this will be achieved by electroplating the radionuclide to a $10-\mathrm{mm}$-diameter circle on a stainless steel disk. The activity will mainly be determined using $4 \pi \beta-\gamma$ coincidence counting and/or liquid scintillation counting methods independent of the MMC measurements.

MMCs with metallic absorbers usually have no possibility to distinguish between particle types; therefore, the detector needs to be shielded from non-radiative emissions of the source. A simple way to achieve this is to use beryllium windows, but especially for low-energy photons the absorption will be too large and hard to estimate with 


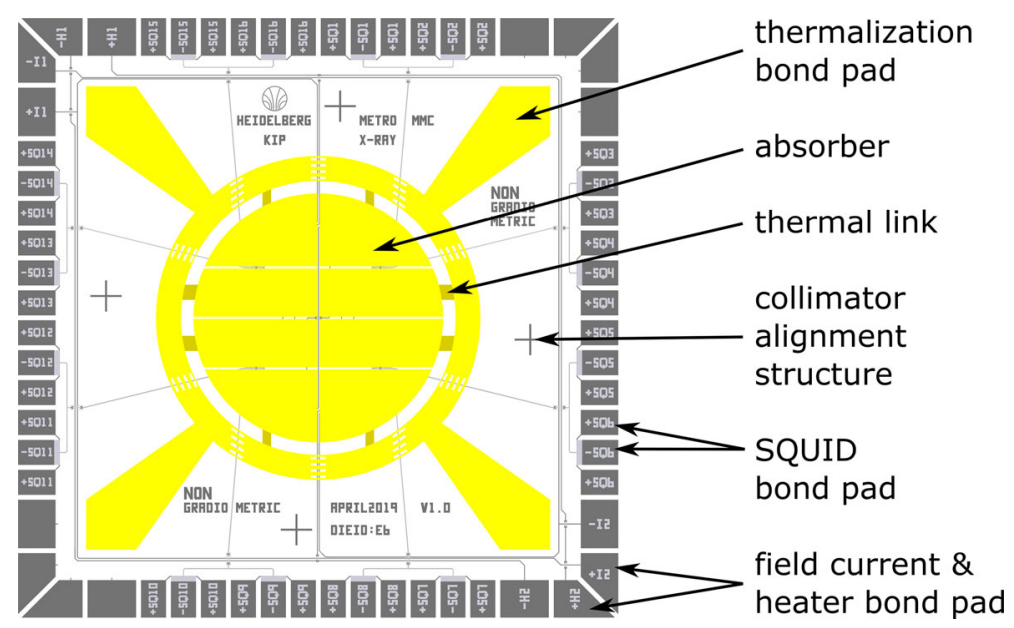

Fig. 1 The new MetroMMC X-ray detector. The detailed features are described in the text (Color figure online)

small uncertainties. Since all non-radiative particles from the decays are charged, magnetic fields will be used to deflect the particles, but special care needs to be taken to shield the detector since both the MMC and the read-out SQUID are susceptible to magnetic interference. A superconducting coil is ideal for this application since the induced magnetic field is not present during cool-down, which allows the use of superconducting shielding, e.g., made from niobium, for the detector, and the field strength on the order of $10 \mathrm{mT}$ can be adjusted to the expected particle energies. The exact operational parameters need be found in simulations and depend on the geometry, which has not been finalized at this time.

A collimator is used to define the available area of the absorber. Especially the edge geometry needs to be designed carefully to avoid scattered particles reaching the detector, while keeping the particle transmission through the collimator edge to a minimum. The design principles are, e.g., well described in [23]. The absorber needs to be characterized for its detection efficiency in the desired energy range, ideally both with simulations and experimentally [24]. In addition, the geometric efficiency needs to be calibrated very precisely, which will be done using a well characterized ${ }^{210} \mathrm{Po} \alpha$-source of the same geometry as the X-ray sources.

For higher-energy X-rays, applicable to ${ }^{109} \mathrm{Cd}$ and ${ }^{125} \mathrm{I}$, the MMC detector system "SMX3" has already been set up, well characterized and used for similar measurements at LNE-LNHB, covering an energy range between 5 and $26 \mathrm{keV}[25,26]$.

The remaining radionuclides emit lower energy X-rays, which require a new detector design developed within this project, with a design drawing shown in Fig. 1. It features a segmented circular absorber with an active area of $3 \mathrm{~mm}^{2}(\approx 2 \mathrm{~mm}$ diameter) with 8 pixels of equal absorber area on a $5 \times 5 \mathrm{~mm}^{2}$ substrate. The optimal gold absorber thickness was determined to be $17 \mu \mathrm{m}$, yielding a detection efficiency of $99 \%$ at $9 \mathrm{keV}$. In addition, the design features crosses for precise alignment of the collimator. 
Each of the 4 read-out channels can be connected to a SQUID on 2 of the 4 chip sides each, allowing a very flexible experimental setup. All 4 channels are connected in series for supply of the field generating current and the persistent current switch, which can be connected on two edges. The thermal layout features an on-chip heat bath, that connects to each pixel with a defined thermal link to provide a thermal decay time of $\approx 10 \mathrm{~ms}$ and uses air bridges, which traverse exposed niobium leads. The energy resolution is expected to be better than $5 \mathrm{eV}$.

\section{Source Preparation}

The preparation of the radionuclide sources, both for calorimetric and X-ray spectrometry, can be just as important as the measurements and data analysis themselves. If not done carefully, the techniques can lead to distortions in the spectral shape [10], cause energy shifts [27] or degrade the energy resolution of the measurement [28]. The widely used techniques are drop deposition from liquid solution and electroplating, while ion-implantation is not as widely available, with each technique having its pros and cons.

In liquid solution, the radionuclides are usually present as dissolved salts or in some cases in the form of organic molecules, with an added inactive carrier to stabilize the solution. When dried out, the residual material can lead to scattering and energy loss in the source or chemically caused energy shifts. On the flipside, drop deposition is the easiest available technique since it can be done manually or advantageously by using automatic microdrop dispensers.

With electroplating, the chemical composition of the nuclide is changed, mostly into metallic or metal oxide/hydroxide form. This avoids some of the problems caused by source preparation, but electroplating is not possible for all materials and requires dedicated tabletop setups, the chemical understanding and dedicated development for each element.

Ion-implantation is by far the cleanest technique, since the nuclide is deposited in atomic form directly into the top layer of the substrate or directly into the detector, that will be used for the measurement. Therefore, none of the chemical bonds or scattering can cause a different detector response. Lattice defects or influences from the host crystal lattice are usually negligible. In addition, bigger ion implanting facilities are equipped with high-resolution mass separators and resonant laser ionization that allow nuclide specific implantation without any other isotopes or neighboring elements [29]. The problem with ion-implantation is that it requires dedicated large-scale facilities and often dedicated development efforts at the facilities to successfully and efficiently conduct the implantation.

Within MetroMMC, both electroplating if possible, as well as drop deposition as a backup are foreseen to be employed for source preparation. Details of the latter preparation technique are discussed in [30]. The aforementioned problems with drop deposition will be addressed by trying to limit the crystal sizes of the residual material by using less material per area, enabled by automatic microfluidic drop deposition or the use of nano-structured gold films as successfully shown in [28]. 


\section{Conclusion}

The MetroMMC project aims to determine fundamental decay data of several EC decaying nuclides. Metallic magnetic calorimeters are a mature technology for the tasks at hand; however, the adaptations necessary to achieve the required metrological precision are challenging. Apart from the careful preparation and execution of the experiments with quality assurance steps whenever possible, MetroMMC uses two separate MMC systems at LNE-LNHB and PTB, respectively. Selected measurements will be repeated and analyzed at both institutes to enable validation of the measurement results.

First measurements on capture probabilities are foreseen to still be conducted in 2019, while X-ray measurements will start in early 2020. The complete set of measurements, divided between LNE-LNHB and PTB, is supposed to be finished by the end of the funding period in mid-2021.

Acknowledgements This work was performed as part of the EMPIR Project 17FUN02 MetroMMC. This project has received funding from the EMPIR program co-financed by the Participating States and from the European Union's Horizon 2020 research and innovation program.

Open Access This article is licensed under a Creative Commons Attribution 4.0 International License, which permits use, sharing, adaptation, distribution and reproduction in any medium or format, as long as you give appropriate credit to the original author(s) and the source, provide a link to the Creative Commons licence, and indicate if changes were made. The images or other third party material in this article are included in the article's Creative Commons licence, unless indicated otherwise in a credit line to the material. If material is not included in the article's Creative Commons licence and your intended use is not permitted by statutory regulation or exceeds the permitted use, you will need to obtain permission directly from the copyright holder. To view a copy of this licence, visit http://creativecommons.org/licenses/by/4.0/.

\section{References}

1. G. Jörg, Y. Amelin, K. Kossert, C.L. Gostomski, Geochim. Cosmochim. Acta 88, 51 (2012). https:// doi.org/10.1016/j.gca.2012.03.036

2. D. Fink, J. Klein, R. Middleton, Nucl. Instrum. Methods Phys. Res. Sect. B 52(3), 572 (1990). https:// doi.org/10.1016/0168-583X(90)90479-E

3. G. Bellini et al., J. High Energy Phys. 2013(8), 38 (2013). https://doi.org/10.1007/JHEP08(2013)038

4. B. Alpert et al., Eur. Phys. J. C 75(3), 112 (2015). https://doi.org/10.1140/epjc/s10052-015-3329-5

5. L. Gastaldo et al., Eur. Phys. J. Spec. Top. 226(8), 1623 (2017). https://doi.org/10.1140/epjst/e201770071-y

6. E. Aprile et al., Nature 568(7753), 532 (2019). https://doi.org/10.1038/s41586-019-1124-4

7. R. Broda, P. Cassette, K. Kossert, Metrologia 44(4), S36 (2007). https://doi.org/10.1088/0026-1394/ $44 / 4 / \mathrm{s} 06$

8. H. Rotzinger, M. Linck, A. Burck, M. Rodrigues, M. Loidl, E. Leblanc, L. Fleischmann, A. Fleischmann, C. Enss, J. Low Temp. Phys. 151(3), 1087 (2008). https://doi.org/10.1007/s10909-008-97875

9. M. Loidl, M. Rodrigues, B. Censier, S. Kowalski, X. Mougeot, P. Cassette, T. Branger, D. Lacour, Appl. Radiat. Isot. 68(7), 1454 (2010). https://doi.org/10.1016/j.apradiso.2009.11.054

10. M. Loidl, M. Rodrigues, C. Le-Bret, X. Mougeot, Appl. Radiat. Isot. 87, 302 (2014). https://doi.org/ 10.1016/j.apradiso.2013.11.024

11. M. Loidl, C. Le-Bret, M. Rodrigues, X. Mougeot, J. Low Temp. Phys. 176(5), 1040 (2014). https:// doi.org/10.1007/s10909-014-1190-9 
12. P.C.-O. Ranitzsch, C. Hassel, M. Wegner, D. Hengstler, S. Kempf, A. Fleischmann, C. Enss, L. Gastaldo, A. Herlert, K. Johnston, Phys. Rev. Lett. 119, 122501 (2017). https://doi.org/10.1103/PhysRevLett. 119.122501

13. M. Loidl, M. Rodrigues, R. Mariam, Appl. Radiat. Isot. 134, 395 (2018). https://doi.org/10.1016/j. apradiso.2017.10.042

14. M. Loidl, J. Beyer, L. Bockhorn, C. Enss, D. Györi, S. Kempf, K. Kossert, R. Mariam, O. Nähle, M. Paulsen, M. Rodrigues, M. Schmidt, J. Low Temp. Phys. 193(5), 1251 (2018). https://doi.org/10.1007/ s10909-018-1933-0

15. X. Mougeot, EPJ Web Conf. 146, 12015 (2017). https://doi.org/10.1051/epjconf/201714612015

16. X. Mougeot, Appl. Radiat. Isot. 134, 225 (2018). https://doi.org/10.1016/j.apradiso.2017.07.027

17. M.W. Haverkort, J. Phys. Conf. Ser. 712, 012001 (2016). https://doi.org/10.1088/1742-6596/712/1/ 012001

18. M. Braß, C. Enss, L. Gastaldo, R.J. Green, M.W. Haverkort, Phys. Rev. C 97, 054620 (2018). https:// doi.org/10.1103/PhysRevC.97.054620

19. A. Fleischmann, C. Enss, G.M. Seidel, in Cryogenic Particle Detection. Topics in Applied Physics, vol. 99, ed. by C. Enss (Springer, Berlin, 2005), pp. 151-216

20. A. Fleischmann, L. Gastaldo, S. Kempf, A. Kirsch, A. Pabinger, C. Pies, J. Porst, P. Ranitzsch, S. Schäfer, F.V. Seggern, T. Wolf, C. Enss, G.M. Seidel, AIP Conf. Proc. 1185(1), 571 (2009). https:// doi.org/10.1063/1.3292407

21. S. Kempf, A. Fleischmann, L. Gastaldo, C. Enss, J. Low Temp. Phys. 193(3), 365 (2018). https://doi. org/10.1007/s10909-018-1891-6

22. M.M. Bé, V. Chisté, C. Dulieu, M.A. Kellett, X. Mougeot, A. Arinc, V.P. Chechev, N.K. Kuzmenko, T. Kibédi, A. Luca, A.L. Nichols, Table of Radionuclides, Monographie BIPM-5, vol. 8 (Bureau International des Poids et Mesures, Pavillon de Breteuil, F-92310 Sèvres, France, 2016). http://www. bipm.org/utils/common/pdf/monographieRI/Monographie_BIPM-5_Tables_Vol8.pdf

23. S. Pommé, Metrologia 52(3), S73 (2015). https://doi.org/10.1088/0026-1394/52/3/s73

24. R. Mariam, M. Rodrigues, M. Loidl, J. Low Temp. Phys. 193(5), 1269 (2018). https://doi.org/10.1007/ s10909-018-2001-5

25. M. Rodrigues, P. Cassette, M.C. Lépy, M. Loidl, Y. Ménesguen, Appl. Radiat. Isot. 109, 500 (2016). https://doi.org/10.1016/j.apradiso.2015.11.090

26. M. Rodrigues, R. Mariam, M. Loidl, EPJ Web Conf. 146, 10012 (2017). https://doi.org/10.1051/ epjconf/201714610012

27. F. Gatti, P. Meunier, C. Salvo, S. Vitale, Phys. Lett. B 398(3), 415 (1997). https://doi.org/10.1016/ S0370-2693(97)00239-6

28. M.P. Croce, M.W. Rabin, V. Mocko, G.J. Kunde, E.R. Birnbaum, E.M. Bond, J.W. Engle, A.S. Hoover, F.M. Nortier, A.D. Pollington, W.A. Taylor, N.R. Weisse-Bernstein, L.E. Wolfsberg, J.P. Hays-Wehle, D.R. Schmidt, D.S. Swetz, J.N. Ullom, T.E. Barnhart, R.J. Nickles, J. Low Temp. Phys. 184(3), 958 (2016). https://doi.org/10.1007/s10909-015-1451-2

29. F. Schneider, K. Chrysalidis, H. Dorrer, C.E. Düllmann, K. Eberhardt, R. Haas, T. Kieck, C. Mokry, P. Naubereit, S. Schmidt, K. Wendt, Nucl. Instrum. Methods Phys. Res. Sect. B 376, 388 (2016). https:// doi.org/10.1016/j.nimb.2015.12.012

30. L. Bockhorn, J. Beyer, K. Kossert, M. Loidl, O. Nähle, M. Paulsen, P. C.-O. Ranitzsch, M. Rodrigues, J. Low Temp. Phys. (2019). https://doi.org/10.1007/s10909-019-02274-8

Publisher's Note Springer Nature remains neutral with regard to jurisdictional claims in published maps and institutional affiliations.

\section{Affiliations}

P. C.-O. Ranitzsch ${ }^{1}$ (D) - D. Arnold ${ }^{1} \cdot$ J. Beyer ${ }^{2} \cdot$ L. Bockhorn ${ }^{1,6}$. J. J. Bonaparte ${ }^{3,4} \cdot$ C. Enss ${ }^{3} \cdot$ K. Kossert ${ }^{1}$. S. Kempf ${ }^{3} \cdot$ M. Loidl $^{5} \cdot$ R. Mariam ${ }^{5}$. O. J. Nähle ${ }^{1} \cdot$ M. Paulsen ${ }^{2,3} \cdot$ M. Rodrigues ${ }^{5} \cdot M$. Wegner ${ }^{3}$ 
$\bowtie \quad$ P. C.-O. Ranitzsch

philipp.ranitzsch@ptb.de

1 Physikalisch-Technische Bundesanstalt (PTB), Bundesallee 100, 38116 Brunswick, Germany

2 Physikalisch-Technische Bundesanstalt (PTB), Abbestrasse 2-12, 10587 Berlin, Germany

3 Kirchhoff-Institute for Physics, Heidelberg University, Im Neuenheimer Feld 227, 69120 Heidelberg, Germany

4 Micro and Nanotechnology Department, Comision Nacional de Energia Atomica, Av. General Paz 1499, (1650), San Martin, Buenos Aires, Argentina

5 Laboratoire National Henri Becquerel, CEA, LIST, Bat. 602 - PC111, CEA-Saclay, 91191 Gif-sur-Yvette Cedex, France

6 Present Address: Institut für Festkörperphysik - Abteilung Nanostrukturen, Gottfried Wilhelm Leibniz Universität Hannover, 30167 Hannover, Germany 